\title{
Adenovirus and intranuclear inclusions in appendices in intussusception
}

\author{
H J Porter, C J H Padfield, L C Peres, L Hirschowitz, P J Berry
}

\begin{abstract}
Aims: To examine the light and electron microscopic features of appendices removed at the time of surgical reduction of intussusception in children; and to confirm that the viral inclusions seen in some of them are due to adenovirus.

Methods: A series of 39 appendices from cases of intussusception and 15 control appendices were reviewed. Light microscopic examination of haematoxylin and eosin stained sections was performed on all of them and one appendix with large numbers of inclusions was examined by electron microscopy. Non-isotopic in situ hybridisation using a biotinylated DNA probe was carried out on sections of appendix from 30 of the cases of intussusception and from the 15 controls.

Results: Light microscopic examination showed viral inclusions in 19 of the appendices from the cases of intussusception and in none of the controls. Electron microscopic examination showed virus particles with the typical features of adenovirus. Most of the appendices with viral inclusions in the haematoxylin and eosin stained sections also contained adenovirus DNA as shown by in situ hybridisation.

Conclusions: Viral inclusions seen in appendices from cases of intussusception are caused by adenovirus. Adenovirus DNA was not demonstrable in appendices from cases of intussusception without viral inclusions and the aetiological factors involved in intussusception in these children remain unknown.
\end{abstract}

(f Clin Pathol 1993;46:154-158)

Bristol Royal Hospita for Sick Children

Bristol

H J Porter

L C Peres

P J Berry

Department of

Histopathology Bristol

Royal Infirmary

Bristol

C J H Padfield

Department of

Histopathology St

Michael's Hospital

Michael's

L Hirschowitz

Correspondence to: Dr H J Porter

Department of Paediatric

Pathology, Bristol Royal

Hospital for Sick Children,

St Michael's Hill, Bristol

BS2 8BJ

Accepted for publication 7 August 1992 tion. The association between intussusception and adenovirus infection was first suggested by Strang, ${ }^{2}$ and following this three different studies showed that adenovirus could be recovered from $50 \%$ or more of children with intussusception, significantly more than in controls. ${ }^{3-5}$ More recently a study by Nicolas et al compared the incidence of adenovirus and rotavirus in 64 children with surgically confirmed intussusception. ${ }^{6}$ Adenovirus was found in 25 of the 64 cases (39\%). Serotypes 1 and 2 were most common (10 cases each), followed by type 5 (three cases) and types 3 and 4 (one case each).

Yunis et al performed a histological and ultrastructural study of 682 appendices of which 30 were from cases of ileocolic intussusception. ${ }^{7}$ These 30 cases were examined electron miroscopically. They described two types of intranuclear changes seen on light microscopy as presumptive evidence of adenovirus infection, namely eosinophilic nuclear inclusion bodies and "smudge cells", in which the nucleus is slightly enlarged, homogeneous, and basophilic. Electron microscopic examination showed clusters of replicating viral particles with a dense central nucleoid surrounded by a capsid, appearances typical of adenovirus. In 11 of the $30(36.6 \%)$ cases viral inclusions were demonstrated by light and electron microscopy. Cultures were obtained in five cases and these showed adenovirus serotypes 2 (three cases), 3 , and 5 (one case each).

The aim of the present investigation was to examine the light microscopic findings in a series of appendices removed from children with intussusception and to confirm the electron microscopic appearance of the viral inclusions.

\section{Method}

Blocks from 39 appendices removed at the time of surgical reduction of intussusception between 1985 and 1991 were retrieved from the files of Bristol Children's Hospital. One haematoxylin and eosin stained section containing tip, middle, and proximal end from each of these cases and from each of 15 age matched controls was examined blind by light microscopy. The control cases were appendices removed incidentally during other surgery for example, for Hirschsprung's disease and intestinal malrotation. The features noted were viral inclusions, epithelial disarray with loss of polarity of the surface epithelium, shedding of the epithelium into the lumen, and lymphoid hyperplasia as indicated by large lymphoid follicles with active germinal centres containing many tingible body macrophages. Electron microscopy was carried out on one formalin fixed appendix with 


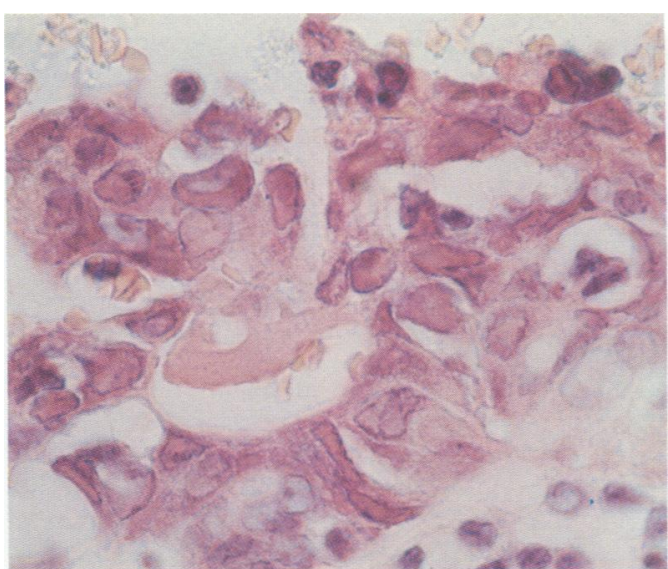

Figure 1 Appendix with epithelial disarray and intranuclear viral inclusions (haematoxylin and eosin).

particularly abundant viral inclusions, which was post-fixed in osmium.

In situ hybridisation for adenovirus was performed on 30 appendices from cases of intussusception (15 with viral inclusions and 15 without inclusions by light microscopy) and on the 15 control appendices. In situ hybridisation was performed using Enzo Pathogene Tissue Preparation Kit and Enzo Pathogene DNA Probe Assay for identification of adenovirus (British distributers Cambridge Bioscience). The biotinylated probe is described as detecting adenovirus serotypes $4,5,7,11,20,40$ and 41 . The kit uses proteinase $\mathrm{K}$ for digestion and $3 \%$ hydrogen peroxide to block endogenous peroxidase. After denaturation at $95^{\circ} \mathrm{C}$, hybridisation occurs at room temperature. The detection system is avidin biotinylated horseradish peroxidase with aminoethyl carbazole as the chromogen. The sections were counterstained with $50 \%$ Harris's haematoxylin for 30 seconds.

For the first batch of sections the positive control for the in situ hybridisation was a slide with HeLa cells infected with adenovirus 5, provided by Enzo. An appendix in the first batch which gave a positive result was used as positive control in subsequent in situ hybridisation procedures. The negative control was a section from the positive appendix treated in the same way as the test sections but with no probe applied to it.

The clinical notes of the 39 children with intussusception were reviewed to determine the results of any virological investigation.

\section{Results}

The children were aged 1 month to 4 years (mean 8.5 months) and all but three were 1 year or under. Table 1 summarises the mor- phological findings on light microscopic examination of the appendices. Viral inclusions were present in 19 of the 39 appendices from cases of intussusception (49\%) and in none of the controls. The number of inclusions varied from a few to hundreds in any one section. Most of the inclusions were of the eosinophilic rather than the "smudge cell" type. Epithelial disarray (fig 1) including budding of the epithelium into the lumen was a consistent feature in appendices with viral inclusions but it was also found in appendices from cases of intussusception without viral inclusions and in the controls, although in these it was less prominent. Shedding of the epithelium into the lumen was present in just over half the appendices with inclusions but in only two without inclusions and in none of the controls. Lymphoid hyperplasia was present in most of the appendices, although it was slightly less common in the controls. Electron microscopic examination of one case with inclusions showed viral particles with the typical appearance of adenovirus (fig 2).

Table 2 shows the results of the in situ hybridisation. The method gave strong positive staining of the inclusions with very little background staining. The nuclei of surface epithelial cells with inclusions stained positively as did cells that had been shed into the lumen (fig 3). There were also scattered positive epithelial cells deeper within the glands (fig 4) and occasional positive cells in lymphoid follicles. Both these last two groups of positive cells were difficult to identify in haemotoxylin and eosin stained sections due to their scarcity. Positive lymphoid cells were present in nine of the 12 appendices that were positive with in situ hybridisation.

Virological studies were performed on only nine of the children with intussusception. Of those without viral inclusions, four patients had virological investigations: adenovirus was cultured from the mesenteric lymph node of one patient, one patient had negative faeces, one a negative throat swab and one a negative rectal swab, mesenteric lymph node, and appendix. Of the five patients with inclusions who had virological investigations, the appendix of one yielded adenovirus type 1 , on culture, although the faeces were negative. In three patients adenovirus was found by electron microscopic examination of the faeces and in one patient the faeces were negative. There was no seasonal variation in the incidence of intussusception or in the cases of intussusception in which adenovirus was detected. There was a large increase in the number of intussusceptions requiring surgical reduction in 1991 (17 cases compared with 22 cases for 1985-1990 inclusive), but no

Table 1 Morphological findings in stained sections of appendices

\begin{tabular}{|c|c|c|c|c|}
\hline & Number & $\begin{array}{l}\text { Lymphoid } \\
\text { hyperplasia }\end{array}$ & $\begin{array}{l}\text { Epithelial } \\
\text { disarray }\end{array}$ & $\begin{array}{l}\text { Epithelial } \\
\text { shedding }\end{array}$ \\
\hline \multicolumn{5}{|l|}{ Intussusceptions } \\
\hline With viral inclusions & 19 & 18 & 19 & 11 \\
\hline Without viral inclusions & 20 & 18 & 11 & 2 \\
\hline Controls & 15 & 11 & 8 & 0 \\
\hline
\end{tabular}




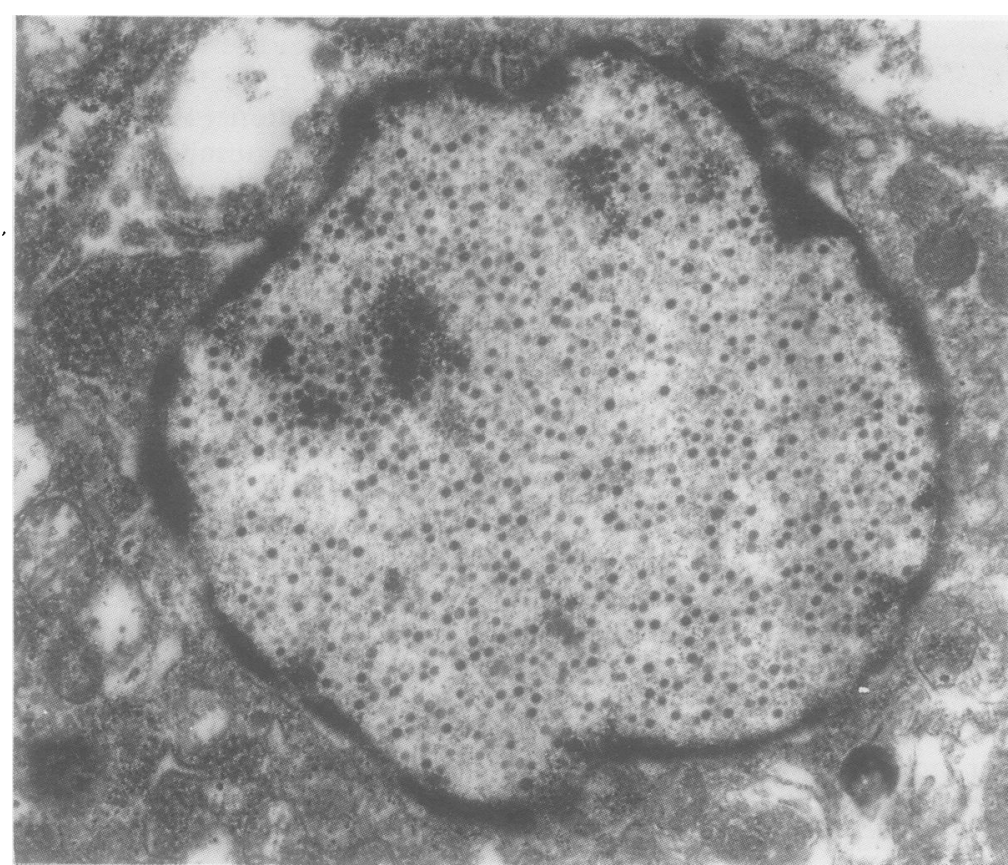

Figure 2 Electron micrograph of epithelial cell nucleus showing typical adenovirus particles.

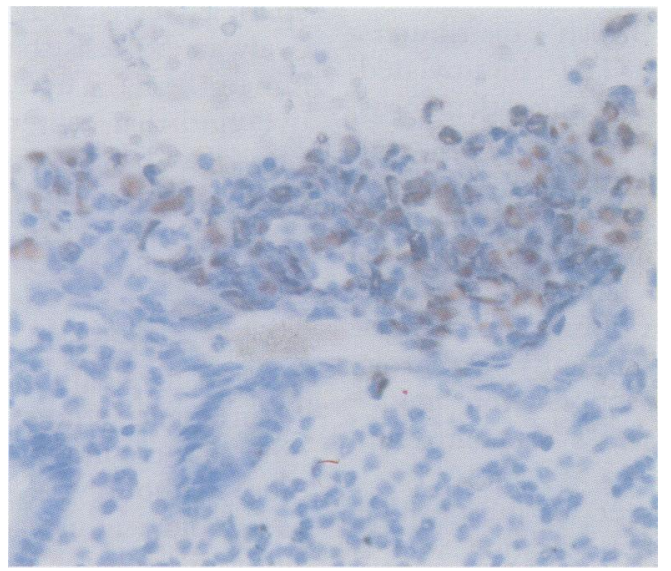

Figure 3 Adenovirus in surface epithelial cells demonstrated by in situ hybridisation.

increase in the proportion with viral inclusions (six out of 17 cases for 1991 and 13 out of 22 cases for 1985-1990).

\section{Discussion}

Intussusception is the commonest cause of intestinal obstruction in young children and still has a substantial mortality. ${ }^{8}$ Adenoviruses are ubiquitous and cause upper respiratory tract infections, conjunctivitis, pneumonia, diarrhoea and mesenteric adenitis. Several clinical and virological studies have shown a relation between adenovirus infection and intussusception. Gardner et al studied 38

Table 2 In situ hybridisation

\begin{tabular}{lll}
\hline & Number & ISH positive \\
\hline With viral inclusions & 15 & 12 \\
Without viral inclusions & 15 & 0 \\
Controls & 15 & 0 \\
\hline
\end{tabular}

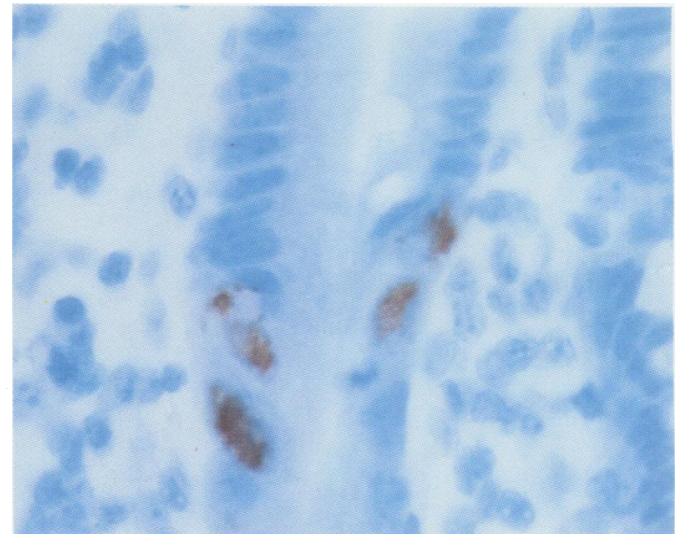

Figure 4 Glandular epithelial cells containing adenovirus demonstrated by in situ hybridisation.

children admitted with intussusception and compared them with 62 children with diarrhoea, 38 with pneumonia and bronchiolitis and 10 normal controls. ${ }^{9}$ Faeces and throat swabs were cultured from all children and mesenteric lymph nodes from 21 of the 38 children with intussusception. Thirty five had paired sera examined. Adenovirus was isolated from the faeces of $46 \%$ of the children with intussusception. When adenovirus was isolated from more than one site in a child it was of the same type and the authors considered that this, together with a positive antibody response in 18 children, indicated active and probably recent systemic infection. Adenoviruses were isolated from the faeces in $3.2 \%$ of children with diarrhoea, $2 \cdot 6 \%$ of those with pneumonia and bronchiolitis, and from $3.7 \%$ of normal children. Two children with intussusception were also found to have herpes simplex virus and one to have polio virus in their faeces. Ross et $a l^{4}$ and Potter performed similar studies and found adenovirus in $61 \%$ and $63 \%$, respectively, of patients with intussusception compared with between $2 \%$ and $6 \%$ of controls. There has been only one large study from the United States which documented the presence of viral inclusions in appendices from cases of intussusception, and suggested that they were attributable to adenovirus. ${ }^{7}$

The finding in the present investigation of viral inclusions in $49 \%$ of appendices from cases of intussusception is higher than that found in the American study but within the range of the British virological studies. There have been no large studies correlating histological appearances with virological investigations. In the series of 30 appendices examined by Yunis et $a l^{7}$ five patients with viral inclusions had virological culture performed on rectal swabs and adenovirus was grown from all of them. In only one of these cases was adenovirus detected in the faeces electron microscopically. The histological diagnosis of adenovirus is obviously subject to sampling error but the incidence in the present investigation correlates well with the incidence found in the larger virological studies. Although there is one case in this study where adenovirus was cultured from mesen- 
teric lymph node even though histology of the appendix was negative, it seems likely that most cases can be diagnosed histologically if the appendix is removed. This study has confirmed the previous reports of a significant association between adenovirus and intussusception in that none of the control appendices had viral inclusions.

The morphological changes of epithelial disarray and shedding are clearly not specific to cases of intussusception associated with adenovirus infection as they were found, albeit to a lesser extent, in cases of intussusception without viral inclusions and in controls. These changes presumably simply represent disturbance of the epithelium which may be due to a number of different causes including the appendix being involved in the intussusception.

The use of in situ hybridisation has demonstrated that the viral inclusions are due to adenovirus in most cases. There are several possible explanations for the three appendices with viral inclusions on haematoxylin and eosin stained sections that were negative with in situ hybridisation. First, of the serotypes usually associated with intussusception, ${ }^{1-6}$ the probe used is described as only detecting four and five so it is possible that the inclusions negative for in situ hybridisation are of serotypes $1,2,3$ or 6 . Serotypes 1 and 2 seem to be the most common and it is therefore perhaps surprising that as many as 12 of the cases were positive with in situ hybridisation because the probe is not supposed to detect these serotypes. Secondly, there may not have been any viral inclusions in the particular sections on which in situ hybridisation was performed. Thirdly, the inclusions may have been caused by another virus. Against this last possibility is the fact that the inclusions in the in situ hybridisation negative cases had the same morphology on haematoxylin and eosin staining as those in the in situ hybridisation positive cases. The finding of viral inclusions in lymphoid cells is not surprising because adenovirus seems to cause lymphoid hyperplasia, can be cultured from mesenteric lymph nodes, and was first isolated from adenoids. However, this does not seem to be well documented probably because the number of lymphoid cells with inclusions is small.

There were no cases positive with in situ hybridisation that were negative for inclusions on haematoxylin and eosin staining, indicating that routine examination of the appendix is at least as sensitive as in situ hybridisation for detecting viral inclusions. Thus in cases of intussusception without viral inclusions adenovirus DNA is not demonstrable by in situ hybridisation. This raises the question of the aetiology of the intussusception in these children which make up about $50 \%$ of the cases.

Konno et $a l^{10}$ suggested that rotavirus may also have a role in the aetiology of intussusception. There were 30 patients in their study and the diagnosis was made on clinical and radiological findings. Faecal specimens were obtained from all and electron microscopy performed. Paired sera were available in 18 cases and complement fixation tests were carried out for rotavirus and adenovirus type 2 . Eleven of the 30 patients shed rotavirus (37\%) compared with eight who shed adenovirus $(27 \%)$. Serological findings showed a four-fold or greater increase in antibodies to rotavirus in six patients including one who did not shed rotavirus in the faeces. Serological evidence of adenovirus infection was present in 11 patients including three who did not shed adenovirus in their faeces. The authors concluded that rotavirus and adenovirus are both associated with intussusception, although there were no controls in the study and no virus isolation was performed.

The more recent study by Nicolas et $a l^{6}$ examined 64 children with intussusception requiring surgical reduction. Virological investigations consisted of virus isolation from rectal and throat swabs and serology for adenovirus and rotavirus. Twenty five (39\%) patients shed adenovirus. Five patients shed other viruses (one polio, three echo, and one herpes simplex). Six patients showed serological evidence of rotavirus infection but in four it was associated with isolation of adenovirus. Unlike Konno et al, these authors consider that rotavirus is not an aetiological agent in intussusception.

Other, viruses reported to be associated with intussusception include herpes virus 6 (three cases), ${ }^{11}$ and $E$ coli $0157: \mathrm{H} 7 .{ }^{12}$

The large increase in the number of operative reductions of intussusception in Bristol in 1991 seems to be the result of an increased rate of failed enema reductions rather than an increased number of cases of intussusception as a whole. The numbers are small but there is no evidence of an increase in the numbers of intussusceptions associated with viral inclusions during 1991. This, together with the fact that the virological studies which included operated and non-operated cases, found a similar incidence of adenovirus infection does not support the possibility that cases requiring surgical reduction are more likely to be associated with adenovirus infection.

In conclusion, by use of in situ hybridisation we have proved conclusively that most of the viral inclusions seen in the appendices from children with intussusception are due to adenovirus. This investigation has shown serotypes 4 and 5 to be the most common in the cases studied, if as is claimed by Enzo, the probe used does not detect serotypes $1,2,3$ and 6 . This is in contrast to the virological studies which generally indicate that serotypes 1 and 2 are the most common. None of the appendices that did not show viral inclusions in sections stained with haematoxylin and eosin was positive with in situ hybridisation, indicating that straightforward morphology is adequate for the diagnosis of adenovirus infection. The role of other possible aetiological factors in adenovirus negative cases of intussusception remains unresolved. 
The Department of Paedatric Pathology is supported by a grant from the Foundation for the Study of Infant Death. LCP is supported by a grant from the Brazilian National Research Council.

1 Potter CW, Zachary RB. The etiology of intussusception. With particular attention to the adenovirus infections. With particular attention to the adenovin North Am 1964;44:1509-20.
Surg Clin

2 Strang R. Intussusception in infancy and childhood. A review of 400 cases. Br $\mathcal{F}$ Surg 1959;46:484-95.

3 Bell TM, Steyn JH. Viruses in lymph nodes of children with mesenteric adenitis and intussusception. $\mathrm{Br} \mathrm{Med} \mathcal{F}$ 1962;2:700-2.

4 Ross JG, Potter CW, Zachary RB. Adenovirus infection in association with intussusception in infancy. Lancet 1962,ii:221-3.

5 Potter CW. Adenovirus infection as an aetiological factor in intussusception of infants and young children. $\mathcal{F}$ Pathol Bacteriol 1964;88:263-73.

6 Nicolas JC, Ingrand D, Fortier B, Bricout F. A one-year virological survey of acute intussusception in childhood. 7 Med Vir 1982;9:267-71.

7 Yunis EJ, Atchison RW, Michaels RH, DeCicco FA. Adenovirus and ileocecal intussusception. Lab Invest 1975;33:347-51.

8 Stringer MD, Pledger G, Drake DP. Childhood deaths from intussusception in England and Wales, 1984-9. Br rom intussusception in England and Wales, 1984-9. Br

9 Gardner PS, Knox EG, Court SDM, Green CA. Virus infection and intussusception in childhood. Br Med $\mathcal{F}$ 1962;2:697-700.

10 Konno T, Suzuki H, Kutsuzawa T, et al. Human rotavirus infection in infants and young children with intussusception. $\mathcal{F}$ Med Virol 1978;2:265-9.

11 Asano Y, Yoshikawa T, Suga S, Hata T, Yamazaki T, Yazaki $T$. Simultaneous occurrence of human herpesvirus 6 infection and intussusception in three infants. Pediatr Infect Dis f 1991;10:335-7.

12 Lopez EL, Devoto S, Woloj M, Pickering LK, Cleary TG Intussusception associated with Escherichia coli 0157:H7. Pediatr Infect Dis $\mathcal{f}$ 1989;8:471-3. 